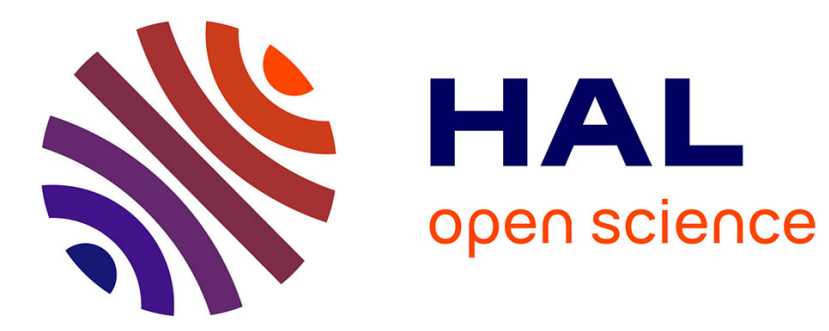

\title{
Empirical Assessment of Multimorphic Testing
}

Paul Temple, Mathieu Acher, Jean-Marc Jézéquel

\section{To cite this version:}

Paul Temple, Mathieu Acher, Jean-Marc Jézéquel. Empirical Assessment of Multimorphic Testing. IEEE Transactions on Software Engineering, inPress, pp.1-21. 10.1109/TSE.2019.2926971 . hal02177158

\section{HAL Id: hal-02177158 \\ https://hal.inria.fr/hal-02177158}

Submitted on 8 Jul 2019

HAL is a multi-disciplinary open access archive for the deposit and dissemination of scientific research documents, whether they are published or not. The documents may come from teaching and research institutions in France or abroad, or from public or private research centers.
L'archive ouverte pluridisciplinaire HAL, est destinée au dépôt et à la diffusion de documents scientifiques de niveau recherche, publiés ou non, émanant des établissements d'enseignement et de recherche français ou étrangers, des laboratoires publics ou privés. 


\title{
Empirical Assessment of Multimorphic Testing
}

\author{
Paul Temple, Mathieu Acher, Jean-Marc Jézéquel
}

\begin{abstract}
The performance of software systems such as speed, memory usage, correct identification rate, tends to be an evermore important concern, often nowadays on par with functional correctness for critical systems. Systematically testing these performance concerns is however extremely difficult, in particular because there exists no theory underpinning the evaluation of a performance test suite, i.e., to tell the software developer whether such a test suite is "good enough" or even whether a test suite is better than another one. This paper proposes to apply Multimorphic testing and empirically assess the effectiveness of performance test suites of software systems coming from various domains. By analogy with mutation testing, our core idea is to leverage the typical configurability of these systems, and to check whether it makes any difference in the outcome of the tests: i.e., are some tests able to "kill" underperforming system configurations? More precisely, we propose a framework for defining and evaluating the coverage of a test suite with respect to a quantitative property of interest. Such properties can be the execution time, the memory usage or the success rate in tasks performed by a software system. This framework can be used to assess whether a new test case is worth adding to a test suite or to select an optimal test suite with respect to a property of interest. We evaluate several aspects of our proposal through 3 empirical studies carried out in different fields: object tracking in videos, object recognition in images, and code generators.
\end{abstract}

Index Terms-software product lines; software testing; performance testing; test evaluation

\section{INTRODUCTION}

On May 7, 2016, a 2015 Tesla Model S collided with a tractor trailer crossing an uncontrolled intersection on a highway west of Williston, Florida, USA, resulting in fatal injuries to the Tesla driver. On January 19, 2017, the NHTSA (National Highway Traffic Safety Administration) released a report on the investigation of the safety of the Tesla autonomous vehicle control system. Data obtained from the Model S indicated that: 1) the Tesla was being operated in Autopilot mode at the time of the collision; 2) the Automatic Emergency Braking (AEB) system did not provide any warning or automated braking for the collision event; and 3) the driver took no braking, steering or other actions to avoid the collision. The conclusion was the investigation did not reveal any safety-related defect with respect to predefined requirements from the system. However, the crash did actually occur. Without questioning the legal aspects that are definitively covered in the NHTSA report, one might wonder why the computer vision program did not "see" this huge trailer in the middle of the road. Of course, a posteriori, it is easy to understand that the Tesla crash videos recorded by Autopilot were not under ideal lighting conditions. Background objects blended into vehicles that needed to be recognized, making it difficult for any computer to process the video stream correctly. On top of that, no wheels were visible under the trailer, which complicated its identification as a vehicle in the middle of the road. More recently, on March 18, 2018, an autonomous Uber vehicle hit a pedestrian crossing a road in Arizona, USA. The pedestrian crossed outside any near crosswalks, at night, on a road where there were no public lighting. A video of the accident was released a few weeks later showing that the vehicle did not even try to dodge or brake before it hits the person and provokes her death. However, the associated released report stated that the system actually recognized the pedestrian just before the accident, showing that the embedded recognition system did not fail per se but was "only" slow to recognize the pedestrian under such conditions. Now taking a software engineering perspective, these situations clearly lead to the usual question from the software testing community: how come that those systems were deployed without being tested under such conditions? This is, of course, partly due to a huge input data space. Going further, since the input space (e.g., videos for testing video tracking) is orders of magnitude larger than typical data, we ask the following set of questions: how much effort should we put in the testing activity? How can we build a "good" test suite? How do we even know that a given test suite is "better" than another one? Structural code coverage metrics for test suites seem indeed 
a bit shaky for that kind of software systems, especially for handling quantitative properties related to performance aspects. This general problem does not only apply to Computer Vision systems. For instance, Generative Programming techniques have become a common practice in software development to deal with the heterogeneity of platforms and technological stacks that exist in several domains such as mobile or Internet of Things. Generative programming offers a software abstraction layer that software developers can use to specify the desired system's behavior and automatically generate software artifacts on different platforms. As a consequence, multiple code generators (also called compilers) are used to transform the specifications/models represented in graphical or textual languages into different general-purpose programming languages such as $\mathrm{C}$, Java, $\mathrm{C}++$, etc. or different byte code or machine code. In this case, from a testing point of view, the input data space is made of models or programs. Defective code generators with respect to performance speed, memory usage, correct identification rate, such as high resource usage or low execution speed, are then hard to detect since testers need to produce and interpret numerous numerical results.

In this paper, we empirically assess the Multimorphic Testing approach which has been briefly introduced in [1], as a method for estimating the relative strength of performance test suites, using a new metric that we call the dispersion score. By analogy with mutation testing, our core idea is to check whether testing different variants implementing the same functionality yields significant differences on the outcome of the tests. These variants are called morphs hereafter. Contrary to functional testing where a clear pass/fail verdict can be used to kill mutants and get a mutation score for the test suite, performance testing can only show performance differences among a set of morphs. With the intuition that a good test suite should be able to highlight outliers among these morphs, we introduce the notion of dispersion score of a test suite to characterize its ability to differentiate morphs of the same system. This work focuses on performance testing and, as such, Multimorphic testing should typically be used after "traditional" functional testing techniques.

Organization of the paper. Section 2 presents our method including the definition of our dispersion score. To show its applicability to various domains, we validate our approach on three different applications in Section 3 (i.e., video tracking, image recognition and code generators). Section 4 discusses some threats of our method and evaluation process. Section 5 presents related work before concluding and proposing future investigation axis in Section 6.

\section{Multimorphic Testing}

\subsection{Motivation}

While functional tests use an Oracle that gives a "pass"/"fail" verdict to check the output conformance of a program, performance testing aims to assess quantitative properties through the execution of software under various conditions. This assessment can be confronted to user-defined requirements, such as "I need a system that is able to process inputs under a fixed amount of time". In the end, the characterization and confrontation process can provide level of insurances about performances of a system. For instance, "From the test I ran, I saw that the system was able to process inputs under 30 seconds in 7 out of 10 cases". One crucial aspect of this process is that the assessment of performances heavily depends on test cases (or given inputs) fed to software systems.

Example 1. For instance, let us consider a Computer Vision (CV) based system designed to detect objects. A key performance indicator could be its precision, defined as the ratio of correctly identified objects with respect to ground truth. Getting a low precision on a given test case (e.g., an image) does not necessarily mean the test case is good and that the CV program is weak, because the test case might simply be too difficult (e.g., a scene with low contrast and poor illumination conditions resulting in objects being barely perceivable). Conversely, if the precision is high, does it mean that the computer vision program is efficient or that the video is simply not very challenging (e.g., just one big, highly contrasted object under ideal illumination conditions)?

Example 2. Let us consider another engineering context. The discovery of (performance) bugs in generators or compilers can be complex. In such a context, what are the most useful test cases considering a test suite that need to be ran in order to find that a generated program using a particular programming language has performance issues such as unexpected high execution time? Once again, we can understand that very simplistic test cases are not interesting as computation times might be too short: taking milliseconds to execute. Conversely, executing unrealistic test cases may result in extremely long execution time or time-out. Here again, we see that what matters is the fact that we are able to discriminate the execution of different programs.

Our general observation is that we cannot assess the performance of a software system solely based on raw and absolute numbers; the performance should rather be put into perspective w.r.t. the difficulty of the task. For doing so, we consider two main axes. First, a software system should be confronted to other morphs in order to establish the relative difficulty of a task. Second, the quality of a test case, and by extension test 
suites, is crucial. A bad test suite may not reveal the underlying difficulty in processing a certain task ${ }^{1}$. We consider a good test case as one being able to discriminate different program implementations based on their observed performances. Overall, we aim to characterize the quality of test suites when assessing quantitative properties of a software system.

\subsection{The principle of Multimorphic Testing}

In this section, we describe how we can associate a score to a performance test suite ${ }^{2}$, based on its ability to discriminate the performance behavior of morphs of the same software system.

The core idea of Multimorphic testing [1] is to evaluate test suites with respect to different morphs being different implementations of the same high-level functionality that should exhibit performance differences. Morphs typically correspond to different parameterizations of a system or to different implementation choices that can be selected at compile time or runtime. For instance, using a specific algorithm for video tracking; or using a particular strategy for a compiler. In these cases, Software Product Line automatic derivation techniques [2], [3] can be used to generate a large number of morphs based on the system variability model.

Said differently and by analogy with mutation testing: Are good test suites able to "kill" weak morphs? Our basic assumption is that a test is "good" when it is able to reveal significant quantitative differences in the performances of morphs. Following the same process as for mutation testing, we derive and exploit morphs (instead of mutants) to reveal significant performance differences (instead of pass/fail verdicts) and eventually assess the relative qualities of test suites.

Our method, called Multimorphic testing, proactively produces morphs that are all tested with the same set of test cases considered as a test suite.

In the example of Figure 1, morphs denoted $M_{1}, M_{2}, M_{3}, \ldots, M_{n}$ are derived thanks to the settings of parameters' values. For instance, in the case of computer vision systems, all morphs implement the same high-level functionality and realize the same task, namely tracking objects in a scene. We use different values for parameters such as Denoise, Confidence, or OpticalFlow, because these can have a significant influence on performance properties such as execution time

1. from our point of view, these tests are pointless but it does not mean that they should be considered as such in the context of functional testing. On the contrary, some of these "bad" tests can be very useful to detect functional bugs.

2. The score of a single test case is then defined as the score of the singleton test suite made of it. or precision. Once morphs are derived, they can be fed with inputs. Inputs are represented by test cases on the left part of Figure 1. Morphs' performances (e.g., execution time) are measured for each pair $\left(M_{i}, T_{i}\right)$. We represented examples of performances in cells of the performance matrix of Figure 1. We remind that those morphs are supposed to be functionally tested and we suppose they are able to perform the high-level functionality they were designed for.

Ultimately, we need a performance measure (or score) that reflects the ability of a test suite to exhibit different performance behaviors among a set of morphs of the same software system.

\subsection{Desired Properties of the measure}

We want the performance measure to have the following properties:

- (P1) non-negativity: the measure associated to a test suite should be $\geq 0$

- (P2) null empty set: the measure associated with an empty test suite is 0

- (P3) measure of subsets: considering 2 test suites $\mathrm{A}$ and $\mathrm{B}$, if $A \subseteq B$, then measure $(A) \leq$ measure $(B)$

- (P4) measure of test suites: considering $n$ test suites $A_{1}, \ldots$, $A_{n}$, measure $\left(A_{1} \cup \ldots \cup A_{n}\right) \quad \geq$ max (measure $\left(A_{1}\right), \ldots$, measure $\left(A_{n}\right)$

While $P 1$ and $P 2$ are rather general descriptive behaviors, $P 3$ and $P 4$ are more specific to our case in particular regarding the behavior of the score when evaluated on association/combinations of test suites. P3 states that if a test suite is included in a larger one, the measure associated to the larger test suite cannot be lower than the measure associated to the first test suite. P4 states that the combination of two or more test suites should not have an associated measure lower than the maximum measure associated to the individual test suites composing the combination of test suites. These two properties stipulate that increasing the size of a test suite should not decrease its associated score.

\subsection{Design of dispersion measures}

The previous properties aim to restrict the design space of candidate measures and in turn eliminate some of them. For example, in this section, we demonstrate that variance, an intuitive and widely used measure, cannot be chosen in our context. We then propose a new measure that we call the dispersion score which does fulfill the wanted properties.

\subsubsection{Variance}

Variance is probably the most commonly used indicator when analyzing the spreading of measures. It computes the difference between elements of a set with the mean value of this set and 


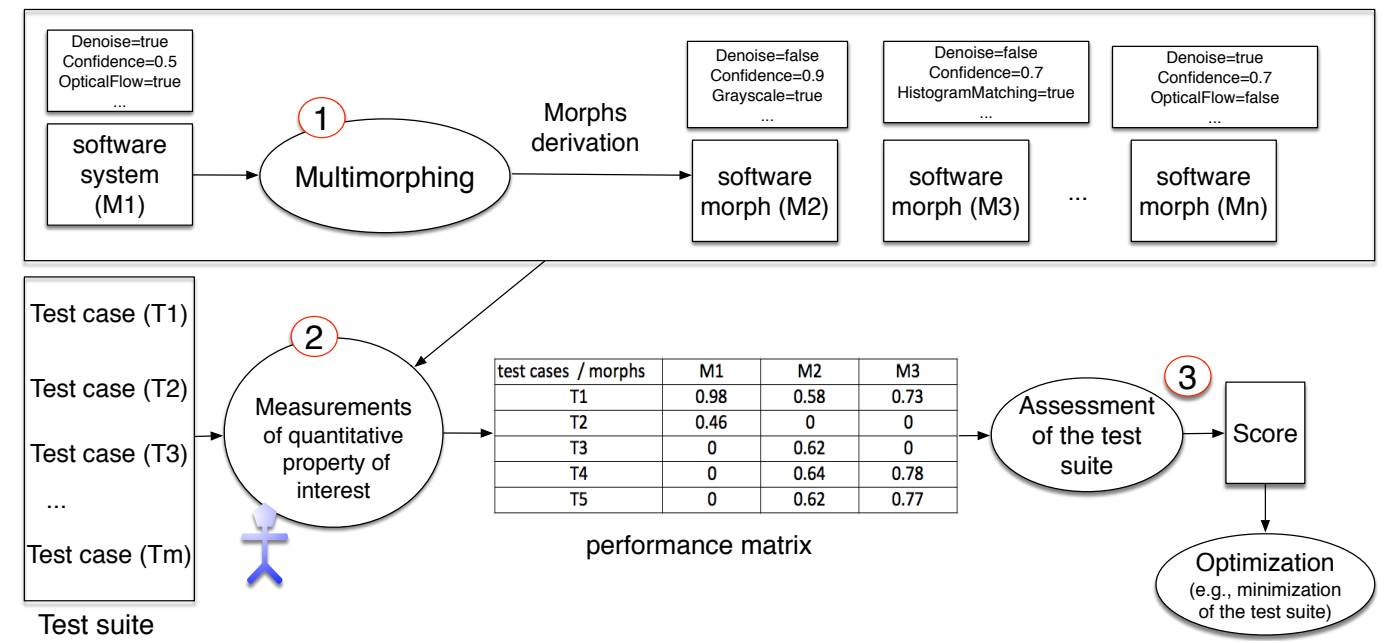

Figure 1: Multimorphic process: morphs are automatically produced (e.g., playing with parameters); for each morph the test suite is executed and performance measurements are gathered; a dispersion score is finally computed to characterize the quality of a test suite

average these differences to produce a value. It is usually described as: $V(X)=\mathbb{E}\left[(X-\mathbb{E}[X])^{2}\right]$ with $X$, a set of observations over a random variable and $\mathbb{E}$, the expected value.

Variance could be interesting but it does not meet the last of our desired properties ((P4) from Section 2.3). We give a counterexample in Table 1: combining two test suites may actually reduce the variance of the resulting test suite. Specifically, Table 1 shows two test suites (Test suite 1 and Test suite 2) both composed of two test cases. Six Morphs are executed on each test suite. Each execution yields a value in the range $[0.1 ; 0.6]$.

Let us compute variances of Table 1 :

- $\quad$ The variance of Test suite 1 is 0.041 ;

- The variance of Test suite 2 is 0.049 ;

- The variance of Test suite 1 and 2 is 0.043 .

The variance of the combination of the two test suites lies in between the two variances of individual test suite. This counterexample shows that (P3) and (P4) are not always met and thus variance cannot be used in our context.

\subsubsection{Dispersion score}

Instead, we propose to use a dispersion score that is inspired from histograms, as they are one of the most popular ways of evaluating the distribution of a continuous variable [4], [5]. An example of histogram based on values given by Test suite 1 of Table 1 is shown in Figure 2.

The observed definition domain (i.e., the range of observed values) is presented on the $X$-axis, while frequency of observations appears on the Y-axis and is defined between 0 and 1 . From left to right, it reads as follows: $16,5 \%$ of observed values lie in the range $[0 ; 0.12], 25 \%$ of observed values lie in the range $[0.12 ; 0.24] \ldots$ and finally $33 \%$ lie in the range $[0.48 ; 0.6]$.

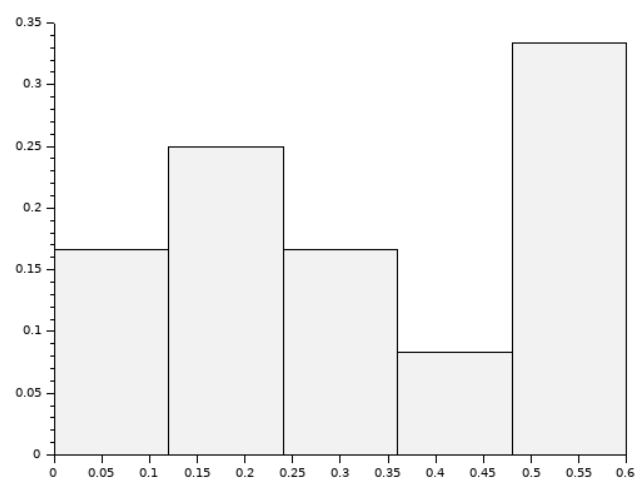

Figure 2: An example of a histogram (based on Table 1).

However, we are not interested in the frequency of each value nor in absolute values but rather in the fact that at least one observation falls into each sub-range (called bin) of the X-axis. We consider a representation that is similar to an histogram since the $\mathrm{X}$-axis defines the definition domain. It is also divided into bins since we want to highlight significant performance differences. The Y-axis however now is just a Boolean value indicating whether at least one performance measure falls into that particular bin. Since we want to "cover" the definition domain, the representation associated to a test suite has to be as dense as possible.

Histograms are parameterized by their number of bins. Bins are defined to gather values which are close from each other (i.e., with insignificant differences). Considering a small number of bins would yield a coarse histogram, gathering values with significant differences. On 


\begin{tabular}{|c|c|c|}
\hline \multicolumn{3}{|c|}{ Test suite 1 } \\
\hline & Test case 1.1 & Test case 1.2 \\
\hline Morph 1 & 0.2 & 0.1 \\
\hline Morph 2 & 0.2 & 0.6 \\
\hline Morph 3 & 0.3 & 0.1 \\
\hline Morph 4 & 0.2 & 0.6 \\
\hline Morph 5 & 0.3 & 0.6 \\
\hline Morph 6 & 0.4 & 0.6 \\
\hline
\end{tabular}

\begin{tabular}{|c|c|c|}
\hline \multicolumn{3}{|c|}{ Test suite 2 } \\
\hline & Test case 2.1 & Test case 2.2 \\
\hline Morph 1 & 0.1 & 0.2 \\
\hline Morph 2 & 0.6 & 0.3 \\
\hline Morph 3 & 0.1 & 0.1 \\
\hline Morph 4 & 0.6 & 0.2 \\
\hline Morph 5 & 0.6 & 0.3 \\
\hline Morph 6 & 0.6 & 0.1 \\
\hline
\end{tabular}

Table 1: An example for showing the inadequacy of variance and illustrating our measure: performance observations gathered for 2 different test suites, each composed of 2 test cases over 6 morphs.

the other hand, too many bins would yield a very fine-grained histogram, providing more details but likely to separate observations with insignificant differences. As a trade-off, we choose to fix the number of bins as the number of morphs to execute $^{3}$. We then define the dispersion score of a test suite as the proportion of activated bins (i.e., non empty bins) in its histogram.

To make this definition more precise, let us consider $n$ morphs and a test suite made of $m$ test cases.

Executing the $m$ test cases on the $n$ morphs yields a matrix $\mathrm{M}$ with $m$ rows and $n$ columns where each cell $M_{i j}$ holds the measured performance value such as precision, recall or execution time, as illustrated in Table 1.

When considering performance properties, it may be difficult to compare different sets of executions: one test might take a few seconds while others could take minutes or more. We thus need to apply a normalization step over these observations. That is, we consider the extrema of observed performances and, for each performance, we apply the following transformation: $\frac{x-\min }{\max -\min }$ where $x$ is an observed performance, min and $\max$ are respectively the minimal and maximal observed performance of a test suite. After this normalization, all performance values $M_{i j}$ are in the range $[0 ; 1]$.

Now, we have to compute the representation, inspired from histogram, for $\mathrm{M}$. The result is a vector $\mathrm{V}$ of size $n$ (since we decided to set the number of bins to the number of morphs) in which each element $V_{i}$ represents whether the corresponding bin is activated or not, i.e. at least one measure $M_{i j}$ falls into it. To do so, we consider the algorithm 1:

The target bin for $M_{i j}$ is the immediate integer larger than $M_{i j} \times n$ (function ceil()), which we set to 1 .

After this step, the vector only contains 0s and $1 \mathrm{~s}$ and the dispersion score is:

$$
\frac{\sum_{i=1}^{n} V_{i}}{n}
$$

3. We admit this choice is rather arbitrary, but empirical assessment already shows interesting results with that choice (Section 3). In fact, finding the best number of bins is a well-known issue [6]. Finding the optimum in our context remains an open question.

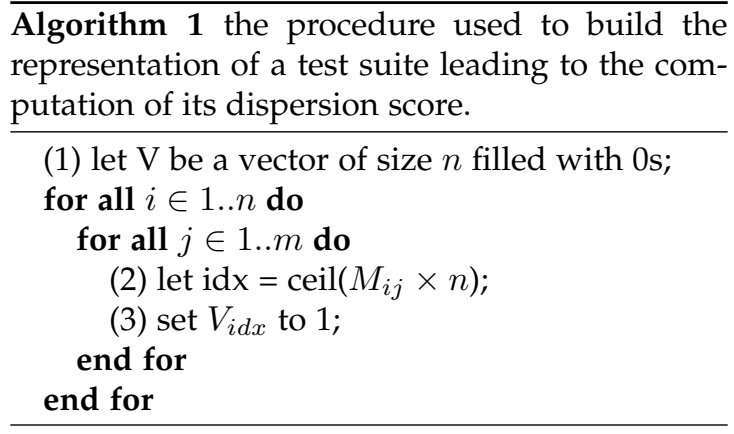

This way, the best possible test suite would activate all the bins of its histogram. That is exactly one observation falls into each bin and yields a dispersion score of 1 , meaning it is able to discriminate every morph.

The worst test suite would have a score close to 0 (exactly $1 / \#$ morphs), because all morphs would behave the same, thus filling only one bin. Dispersion score is thus defined in $[0 ; 1]$ satisfying the first desired property defined in Section 2.3.

The dispersion score of an empty set (i.e., when no test suites are given) is 0 which satisfies the second property. When trying to combine test suites, the fact that we only care about activated bins to compute dispersion scores ensures that $P 3$ and $P 4$ are satisfied.

\section{Empirical Evaluation}

We have introduced the Dispersion Score as a new measure to assess the relative merits of performance test suites. In the following, we want to empirically show that this measure is right, and that it is a right measure.

\subsection{Research questions}

Is the measure right? To be right, the Dispersion Score must at least yield different scores for different test suites, i.e., reflect their ability to exhibit varying performances among of a set of morphs.

We also expect sufficient stability w.r.t. the exact set of morphs used for the measure; so we evaluate the stability of the dispersion score via a sensitivity analysis. Finally, we propose an analysis of the evolution of the dispersion score with respect to the choice of number of bins. 
Is it a right measure? Here, we want to assess the correlation between the actual (relative) effectiveness of performance test suites and their dispersion scores. Depending on the domain, this question will take several very different forms, from helping to select test cases able to reveal performance bugs in code generators, to reducing a test suite without loosing its ranking capabilities.

\subsection{Evaluation settings}

To answer these questions, we applied our method on several application domains and evaluate its results. For each case, we detail: i) what are morphs; ii) what are test suites; iii) how performance measurements are retrieved and used; iv) how we perform the evaluation. Table 2 summarizes the cases; we can notice different scales both in terms of available morphs and test suites.

\begin{tabular}{|c|l|l|l|}
\hline Case & App. Domain & $\#$ morphs & $\begin{array}{l}\# \text { test } \\
\text { suites }\end{array}$ \\
\hline OpenCV & Tracking in videos & 252 & 49 \\
\hline COCO & Obj. rec. in images & 52 & 12 \\
\hline Haxe & Code Generation & 21 & 84 \\
\hline
\end{tabular}

Table 2: The three case studies

\subsubsection{OpenCV case (object tracking in videos)}

In the field of video tracking, the use of large test suites helps building confidence in the robustness of a system and its capability in performing well under various conditions. However, are all those test suites necessary? We consider OpenCV ${ }^{4}$, a popular library, written in $\mathrm{C}++$, implementing different techniques for tracking object of interest in videos.

Morphs. By reverse-engineering a part of OpenCV and using the feature modeling formalism [7], [3], [2], [8], we have elaborated a variability model (including constraints) to formally specify the possible values of parameters and their valid combinations. From this variability model, we automatically sampled 212 configurations by assigning random values to functions' parameters. The configurations are valid w.r.t. the variability model and are used to derive 212 morphs.

Test suites. We use a set of 49 synthetic video sequences. Each video is considered as a test suite on its own composed of only one test case: the video playing the role of the test input data and its ground truth playing the role of the oracle. Videos have been obtained using an industrial video generator [9], [10]. Videos are all different either in the composition of the scene (presence or not of objects we do not want to track such

4. https://opencv.org/ as tree leaves) or in the visual characteristics of the scene (different illumination conditions; presence of heat haze and/or noise). Importantly, a ground truth is automatically generated along videos stating the position of every encrusted objects in every video images such that we can assess the ability of our programs to track objects of interest.

Measurements. Following the Multimorphic method (see Figure 1), we execute all 212 morphs on the 49 videos. For each execution, we measure several quantitative properties such as: Precision, Recall, the execution time and the CPU consumption to cite a few. Precision and Recall measures are ratios given in the range $[0 ; 1]$. They are measured by comparing objects' positions computed by morphs to the generated ground truth. Positions are usually defined by bounding boxes that surround objects. Then, we considered an object as being detected if the intersection between the bounding boxes retrieved by a morph and the one defined by the ground truth is not empty. The execution time is given in seconds while the CPU consumption is expressed as a percentage of one CPU core usage (if the computations are distributed over multiple CPUs then this measure can be higher than $100 \%$ ). Note that to stay within realistic computation boundaries, we set a time-out for every execution we have launched. If an execution exceed this amount of time, its process is killed and its measures are reported as values showing that it has failed (high CPU and memory consumption, zero Precision and Recall measures, etc.). We have considered 13 different quantitative properties for each execution. This yields a total of $212 * 49 * 13=135,044$ performance measures.

Evaluation. Since our method yields a dispersion score associated to a test suite, we evaluate that a test suite created to maximize the dispersion score is actually a "better" test suite than random ones with lesser scores.

\subsubsection{COCO case (object recognition bench- marks)}

For many years, the computer vision (CV) community has been building large datasets that are used as benchmarks [11], [12], [13], [14] in competitions to rank competing image recognition techniques. Here we use the Microsoft COCO dataset on which CV competitions are conducted every year since 2014. Results of competitions are presented on the leaderboard webpage ${ }^{5}$. COCO competitions address different tasks such as detection of objects or segmentation of images. Our evaluation focuses on object detection.

Morphs. Even if we do not know much about the specific details of techniques used by competitors, we know that they are all designed to

5. http:/ / cocodataset.org/\#detections-leaderboard 
recognize objects in images, which means that they can play the role of morphs when applying our Multimorphic method. It should be noted that, for this case, morphs have not been obtained by parameterization - simply, there are existing competing systems realizing the same task and potentially exhibiting performance differences. While those competing systems are implemented in various different ways, and even written in completely different programming languages, we still consider them as morphs because they provide the same high-level functionality (i.e., recognize objects of interest in images) and they are able to process the same inputs and produce outputs that are structured in the same way so that the competition's server is able to deal with them.

Test suites. Competitions using COCO datasets have been running for several years now and each year brings its own dataset. We focus on the 2017 challenge that ended in late November 2017. The dataset is composed of more than 160,000 images. 80 different object classes are specified and more than 886,000 object instances can be detected. Object classes are gathered into concepts. For instance, classes "dog", "giraffe" or "horse" are gathered under a concept called "animal". Similarly, "hot dog" or "carrot" are gathered under the "food" concept. 12 such concepts have been created in total.

To conduct the competition, organizers have decided to split the dataset into two main subsets: first, the set given to competitors along with associated ground-truth so that they can train their models and also perform a validation step. This subset is composed of 120,000 images. The second set is also given to competitors but without associated ground-truth. It is composed of the remaining images and is used to evaluate competitors and thus to establish their ranking. We define each concept as a test suite constituted of images of this second set. All images associated to a concept contain at least one occurrence of this concept. We assume that all concepts are equally represented among this second set yielding to $\frac{40,000}{12} \simeq 3333$ images per test suite.

Measurements. In this study, we focus on the Average Precision performance measure ${ }^{6}$ available for each technique on the leaderboard. This measure is computed over the second set of images and corresponds to the overlap of bounding boxes from a CV technique (i.e., a morph) and the ground truth only known by the server.

The process is the following: (1) each morph is executed on the test suite, generating an output for each test case (i.e., image); (2) all outputs of a morph are sent to the server following a format specified by organizers; (3) for each test case, the

6. Note that we could have considered other measures, it would not have affected our method server computes the overlap of the outputs of a morph and the ground truth; (4) based on the overlap, performance measures are updated; (5) once performance measures are all up-to-date, ranks are computed.

Even if we could have presented results for all 80 object classes, we decided to focus on the 12 concepts for the sake of compactness and exhaustive presentation. However, the method is not changed and conclusions that we present hereafter are similar considering 12 or 80 items.

Evaluation. We consider the ranking computed by the server as the ranking of reference which is available online via the leaderboard webpage. Using our dispersion score to rate test suites, we will try to reduce the number of test suites (i.e., concepts) needed to evaluate competitors' techniques. As a side effect, reducing the number of test suites will also reduce the number of test cases and thus the number of images to gather and annotate. Then, we assess the capability of such a reduced test suite to yield a ranking similar to the original one.

\subsubsection{Haxe case (code generator)}

Today's modern generators or compilers, such as the GNU C Compiler or gcc, become highly configurable, offering numerous configuration options (e.g., optimization passes) to users in order to tune the produced code with respect to the target software and/or hardware platform. Haxe is an open source toolkit ${ }^{7}$ for crossplatform development which compiles to a number of different programming platforms, including JavaScript, Flash, $\mathrm{PHP}, \mathrm{C}++, \mathrm{C \#}$, and Java. It involves many features: the Haxe language, multi-platform compilers, and different native libraries. Moreover, Haxe comes with a set of code generators that translate the manually-written code in Haxe language to different target languages and platforms. One of the main objectives of Haxe is to produce code that has better performances than a hand-written one [15]; it shows the importance of performance aspects of the code generator.

Morphs. Based on previous works presented in [16], [17], we selected 4 popular target languages namely $\mathrm{C}++, \mathrm{C \#}$, Java, PHP. Then, we tuned code generators according to several optimization parameters they provide. More specifically, regarding $\mathrm{C}++$, we chose to apply the different optimization levels available via gcc compiler (O0, O1, O2, O3, Ofast and Os). Regarding other languages, we derived different code generators by toggling different parameters such as dead code elimination, whether use methods in-lining or even the use of code optimizations. For each of the generated variants in one target language, we modified one of these parameters; others are

7. With about 2500 stars on GitHub in June 2018. 
set to default values. In total, we considered 21 different configurations of the Haxe code generator across the four target languages constituting our set of morphs.

Test suites. We reused the same 84 test suites from [16], [17]. Each test suite is composed a number of test cases ranging from 5 to 50 .

Measurements. We used the same testing environment as described in [16], [17], running the same test suites across our 21 morphs, focusing on one property of interest, namely: execution time. We thus collected data relative to the execution time of each generated program. To mitigate the fact that measures could vary because of external factors such as warm-up, or the charge of CPUs, we executed each test case several times on each morph (see [17] for details). Raw measures have been transformed and normalized as follows: (1) Find an execution of reference for each test case and set it to 1 . The reference execution is defined as the one optimizing the considered quantitative property (e.g., minimizing execution time); (2) expressing other observations relative to this test case as a multiplicative factor of the execution of reference. For instance, let us consider two moprhs and a single test case. Assuming the property of interest is execution time, if one execution gives an observation of 35 and the other one of 70 , the first one is the execution of reference and is thus transformed into 1 while the second execution becomes 2 as it took twice the time to be executed. Such transformation has no impact on our proposed solution, since we are not interested in the actual values, but their dispersion. After that first step, we carried on performing a normalization in the range $[0 ; 1]$ to build our representation and compute dispersion scores as explained in Section 2.

Evaluation. Here our criteria will be whether the dispersion score is helping us to select test suites able to reveal performance bugs in code generators.

Presentation of results. Hereafter, we present results for each research question using the three case studies, showing that the same method can be applied to various domains.

\subsection{RQ1: Is the dispersion measure right?}

\begin{tabular}{|c|c|c|c|c|}
\hline Videos & Prog. 1 & $\ldots$ & Prog. 212 & Dispersion \\
\hline \hline vid 01 & 0.683228 & $\ldots$ & 1 & 0.203 \\
\hline \hline & \multicolumn{4}{|c|}{$\ldots$} \\
\hline vid 35 & 0.000396 & $\ldots$ & 0.001709 & 0.118 \\
\hline & \multicolumn{4}{|c|}{$\ldots$} \\
\hline vid 49 & 1 & $\ldots$ & 0.177966 & 0.080 \\
\hline
\end{tabular}

Table 3: Sample of observations for Precision on the OpenCV case

Table 3 shows a representative excerpt of Precision measures that were observed considering the OpenCV case. Similar tables for remaining examples are available in Appendix A. In this table, rows represent test suites and columns are different morphs. As stated before, we used 49 test suites that were executed on 212 morphs. Each cell of the table reports the performance measure of the execution of the program on the video. Based on all retrieved performances for each video, we computed a dispersion score for each individual video which is presented in the last column.

\subsubsection{Can different dispersion scores be ob- served?}

In the following, we want to validate the fact that we can observe variations in performances inducing different dispersion scores.

OpenCV case. Computed dispersion scores range from $\frac{17}{212} \simeq 0.08$ up to $\frac{44}{212} \simeq 0.207$. Over all 49 test suites, the mean value of dispersion scores is $\simeq 0.145$ and the standard deviation $\simeq 0.034$. While these numbers are rather low (i.e., less than a quarter of the bins are activated), it seems that behaviors of all 212 morphs are not equivalent as shown in Table 3. Meaning that not all morphs give the same performance when executed on the 49 videos $^{8}$. For instance, Prog. 1 from Table 3 performs very well on the last video while Prog. 2 is unable to detect anything.

COCO case. In this case, dispersion scores are larger as shown in Table 5. Scores range from 0.308 to 0.423 . Among all 12 concepts, the mean value of dispersion scores is $\simeq 0.359$ and the standard deviation $\simeq 0.040$. Performances of competitors over each concept are available directly on the online leaderboard. Concepts "Electronic" and "Sports" are tied with the maximum dispersion score of 0.423 , concepts "Indoor" and "food" are also tied but they are associated with the lower minimum score of 0.308 .

Haxe case. Dispersion scores from Table 6 range from $\frac{1}{21} \simeq 0.047$ to $\frac{3}{21} \simeq 0.143$. Over all 84 test suites, the mean value of dispersion scores is $\simeq 0.0567$ and the standard deviation $\simeq 0.0202$. These scores are low, showing consistent measures and insignificant differences in the observations. However, those numbers do not indicate the quality of test cases per se. In fact, most of dispersion scores show that only one or two bins are activated in associated histograms. In the case where two bins are activated, we assume that retrieved observations lie close to the separation between the two bins. Variations make observations fall sometimes on one side of the separation and sometimes on the other side. Only one test suite, called the core test suite, is associated with the maximum dispersion score.

8. We obtained similar results for the other quantitative properties we have measured such as recall or performance. 
In this test suite, three bins are activated due to variations. We will investigate further this issue in the next research question.

Taking a step backwards, from the different examples we analyzed, variations in performances can be shown and captured by the use of dispersion score. Every test suite can give a dispersion score that is more or less high depicting how different morphs can perform differently on the same test suite. We point out the fact that absolute values of the dispersion score are meaningless. Comparing scores given by the $\mathrm{COCO}$ case or by Haxe is non-sense as the two cases have nothing in common (i.e., not the same set of morphs, not the same test suites). What is interesting is only the order provided by dispersion scores when ranking test suites according to their dispersion score, as discussed in RQ2 Section 3.4.

\subsubsection{Is dispersion score stable and sensitive to the set of morphs?}

Our hypothesis is that the dispersion score associated with test suites only slightly changes depending on morphs considered in the set that is used. In other words, we want to perform a sensitivity analysis about the dispersion score. This analysis is important as it shows that the dispersion scores are not totally dependant on the choices of the morphs. If dispersion scores vary too much when only one morph is used or removed then the dispersion score is pointless since from one run to another, differing in only one change in the set of morphs, the relative importance given to test suites via dispersion scores would be completely different.

To conduct this experiment, we randomly remove some morphs out of the original pool. That is, we only build dispersion scores taking into account the measures coming from remaining morphs. Taking back the OpenCV case: at each iteration $i t \in 0$.. $\frac{\# \_u s e d \_m o r p h s}{2}$, we remove it morphs from the original set of morphs and observe the effect on dispersion scores for each 49 videos. The whole process of selecting up to half the morphs and computing dispersion score is repeated 50 times in order to flatten the impact of random choices in the removal of the morphs. Algorithm 2 describes how measures have been retrieved.

OpenCV case. Figure 3 shows the evolution of the dispersion score of the videos with the best and worst score depending on the number of morphs that have been removed. On this figure, the $\mathrm{X}$-axis represents the number of morphs that have been removed (from 0 to 106) and the Yaxis represents the associated dispersion scores. Six curves are plotted:

- the three top curves represent results for the video providing the best dispersion score;

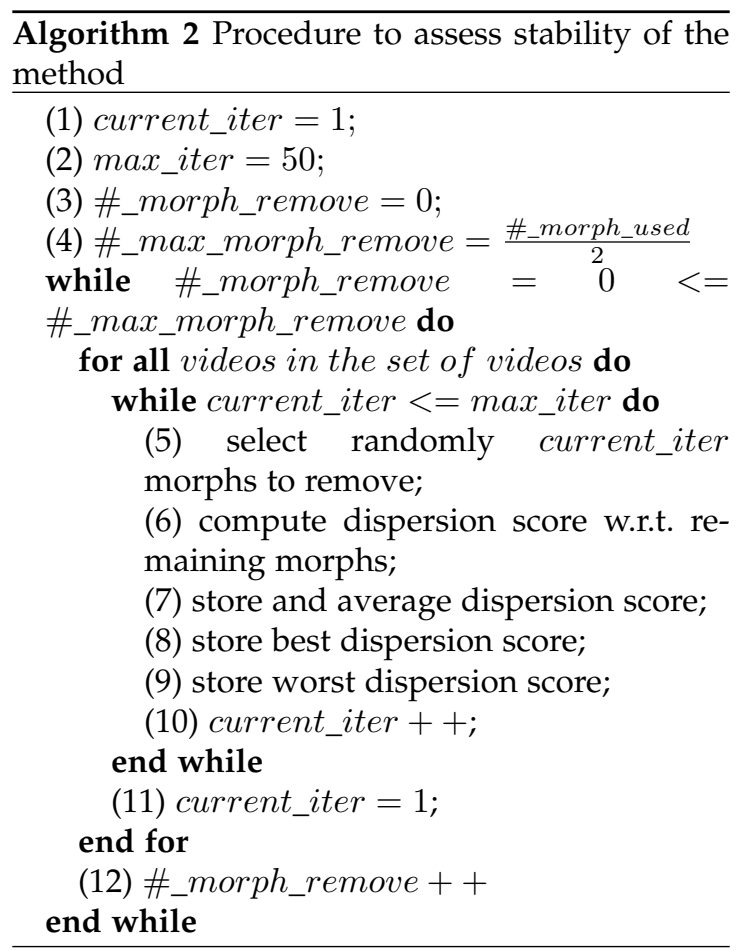

- the three bottom curves represent results for the video with the worst dispersion scores.

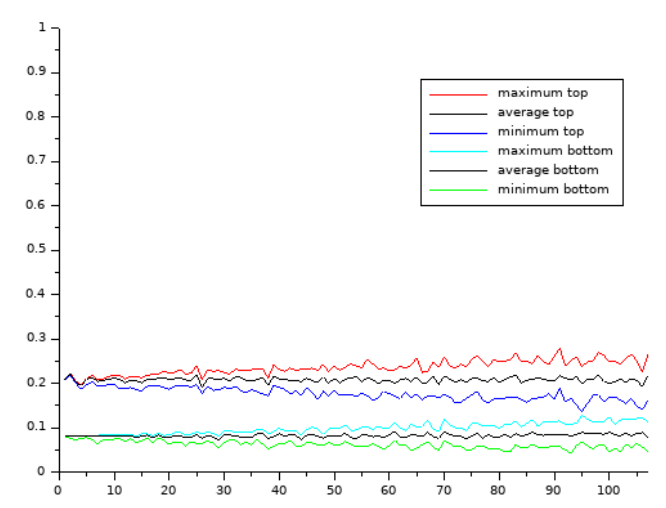

Figure 3: Stability results for the property Precision; X-axis: number of morphs removed; Y-axis: dispersion score

Specifically, considering the best video: (1) The curve in the middle, called average top, reports the average of the dispersion scores, and is obtained through line 7 of Algorithm 2; (2) the top curve, called maximum top, reports the maximum dispersion score using line 8 of Algorithm 2; (3) The so-called minimum top reports the minimum dispersion score as defined by line 9 of Algorithm 2. We depict the same curves for the worst video.

The average curves, namely average top and average bottom are very stable. The four others 


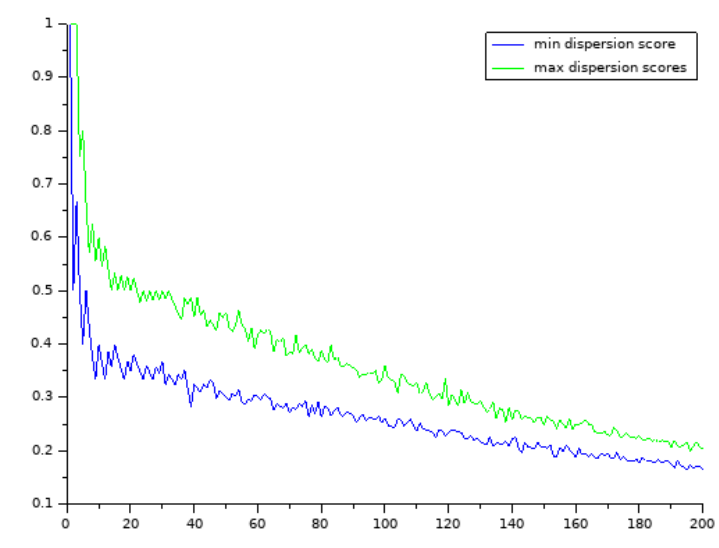

Figure 4: The evolution of minimum and maximum dispersion scores according to the number of bins (COCO case)

(maximum top, minimum top, maximum bottom and minimum bottom) tend to be noisy once fifty morphs or more have been removed. However, despite these variations, none of the curves between the top plots and the bottom ones cross each other. Overall, the results show a stability and consistency in their positions ${ }^{9}$.

COCO case We apply the same process on the COCO dataset but because there are only 52 morphs available, we remove up to 26 morphs instead of 106 which corresponds to remove half our observations. Conclusions are similar to the previous case and dispersion score remains stable and consistent in their positions.

Haxe case We run again the same sensitivity analysis over Haxe. For this case, as we only have a small number of morphs, we remove only up to 10 morphs over the 21 available. Retrieved curves are stable since plateaus appear. Similarly to previous cases (e.g., Fig. 3), top curves and bottom curves never interchange.

\subsubsection{How does the dispersion score evolve w.r.t. to the number of bins?}

Now that we have assessed the stability of the dispersion score according to the morphs, we can also analyze how it evolves depending on our choice for the number of bins. We focus on the $\mathrm{COCO}$ case but conclusions we draw from this case can be generalized to the two others.

Figure 4 shows the evolution of the dispersion scores associated to test suites that have the minimum (bottom curve) and maximum (upper curve) observed dispersion scores. On the X-axis are the number of bins that we used to compute dispersion scores. We make this number vary

9. Again, similar observations can be made for other quantitative properties (e.g., Recall, performance or even a composition of different properties). from 1 to 200. As a remainder, until now, we have used a rule of thumb which is fixing the number of bins to the number of available morphs. In the COCO case, that was 52 because we had 52 morphs. We also remind that the dispersion score is a ratio from the number of activated bins to the total number of bins. We see that dispersion scores tend to decrease as the number of bins increase. This is due to the ratio: as the number of observations is not increasing, growing the number of bins tends to reduce the result. What is interesting here is that the curves tends to join as the number of bins keep increasing. Indeed, because of the definition, if the number of bins tend towards infinity, all dispersion scores tend towards 0 which makes our dispersion score meaningless. On the other side of the figure, using a small number of bins makes the dispersion scores higher (but their absolute values are meaningless) and the differences between the minimum and maximum are exacerbated since the minimum dispersion score drops quickly while the maximum dispersion score may stay high a little longer. However, using a low number of bins makes little sense as we want to capture significant differences in morphs' performances. Indeed, using a small number of bins will create wide bins which would potentially gather significant different performances into the same bin. In Figure 4, it looks like from 20 bins the slope tends to be less abrupt which suggests that picking any number from this point might be a good choice. We would advice to avoid choosing a number of bins that is over 140 as the two curves begin to be very close to each other. We are not able to give a straight, clear, general manner to set the number of bins but, in this specific case, we think that any pick in the range $[20 ; 140]$ might be a good fit. The use of the number of morphs (52) falls into this range and appears to be a reasonable choice for COCO. The sensitivity analysis reported in Figure 4 for $\mathrm{COCO}$ has also been performed for OpenCV and Haxe. Empirical results confirm that number of morphs is relevant, since it lies within the range of suitable number of bins (supplementary material is available online, see Section 3.6).

\subsection{RQ2: Is the dispersion score a right mea- sure?}

We showed that our dispersion score was able to "rate" test suites with different scores. In the following, we would like to evaluate whether those scores have the wanted intuitive meaning: is a test suite with a higher score really better than one with a lesser score?

For addressing this qualitative question, we first used an exhaustive search to create an optimal test suite combining exactly 5 test suites for each of our 3 cases studies. Optimality is 


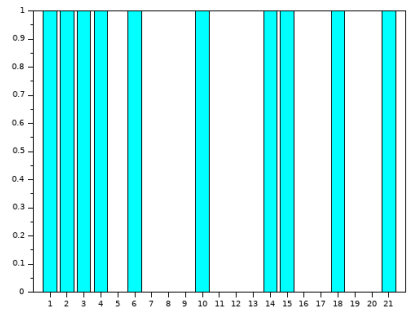

Figure 5: The number of bins activated with the original test suite (composed of 84 test cases). The $\mathrm{X}$-axis represents the index of the bins.

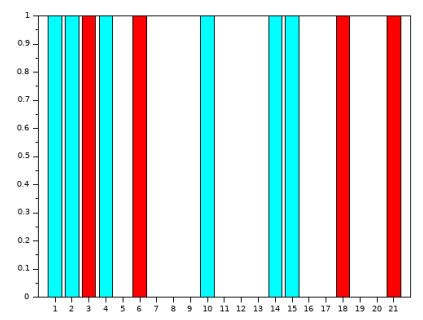

Figure 6: The number of bins activated with the smaller test suite composed of 5 test cases that maximizes the dispersion score. The $\mathrm{X}$-axis represents the index of the bins. Bars in red are bins that are activated with the original test suite showed in Figure 5 but that we fail to activate with our smaller test suite.

defined as maximizing the dispersion score of the new test suite. We then relied on domain specific ground truth to assess whether these "optimal" test suites were indeed any good.

Since the newly built test sets of 5 test suites maximize the dispersion score, we can compare our histogram-based representation from the original test set to this one and observe how far they are one to the other. For instance, taking the Haxe case, the histogram of the original test set using 84 test suites is presented in Figure 5. Blue light bars represent activated bins. Figure 6 shows the histogram of the second test set. Red bars represent differences with Figure 5 in activated bins.

Only 4 bins are different between the two histograms, namely bins 3, 6, 18 and 21. Meaning that, at most, 4 test suites are needed, in the reduced test set, to retrieve the same histogram as the original one. In the end, with those hypothetical 9 test suites, we ensure to retrieve the same diversity in the observed quantitative properties but drastically diminishing testing efforts.

3.4.1 (OpenCV case) Can we create a "good" test suite that is able to differentiate morphs that perform well from others?

The selected "optimal" test suite combining 5 test suites was associated with a score of $\simeq 0.6$ acti- vating 127 bins over 212 in total. In this regard, this "optimal" test suite is at least 3 times better than any individual test suite. But is it really any good?

To answer this question, we have asked a CV expert to cherry-pick twelve new morphs in such a way that six of them are expected to perform well on average and six others would be likely to perform poorly/moderately well. Note that we did not ask the expert to choose very bad configurations that would not recognize anything: since any test suite would be able to tell that they are bad; that would tell us nothing about the relative merit of our test selection process.

Then we ran these 12 morphs on the test suite of 5 videos. For each of these morphs, we plot in Figure 7 the obtained Precision averaged over the 5 executions. The supposedly 6 moderately poor morphs correspond to index 1 to 6 on the X-axis while indexes 7 to 12 correspond to the 6 others supposed to perform well.

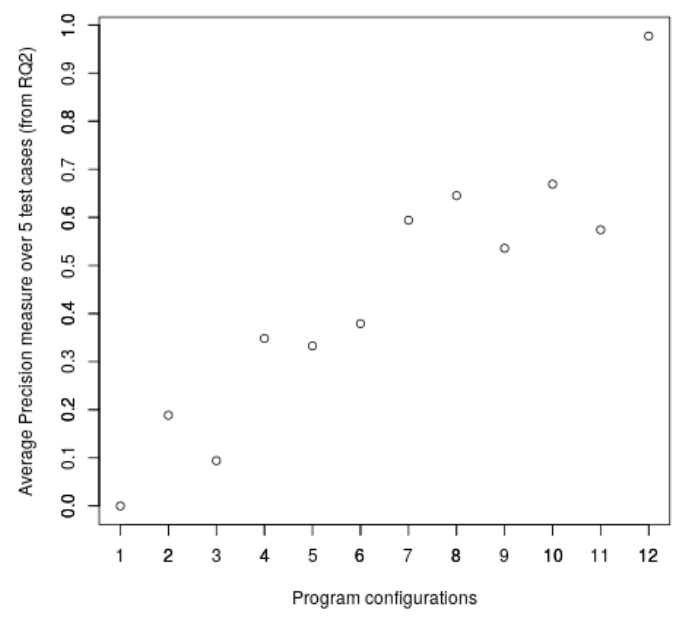

Figure 7: Average Precision measures over the 5 videos from RQ2. On X-axis are the CV morphs: first, the 6 first morphs that are supposed to perform moderately badly; the 6 last morphs are supposed to be good. Y-axis reports the averaged Precision measure over the test suite.

From Figure 7, two classes of programs can be identified: programs which have an average score below 0.4 and programs which reach an average score above 0.5 . This separation corroborates the expert's classification since programs which reach an average Precision above 0.5 (respectively below 0.4) are exactly the ones expected to perform well (respectively moderately poorly). Even if our selected test suite is probably not the best possible one (it is just the best set of exactly 5 test suites), it is already quite able to tell good from poor configurations apart. But is this test suite really better at that than any random 


\begin{tabular}{|c|c|}
\hline Concepts & Dispersion score \\
\hline \hline accessory & \\
\cline { 1 - 1 } animal & \\
\cline { 1 - 1 } appliance & \multirow{2}{*}{0.673} \\
\cline { 1 - 1 } electronic & \\
\cline { 1 - 1 } food & \\
\hline
\end{tabular}

Table 4: The 5 concepts that maximize the dispersion score

test suite of size 5 ? To answer this question, we created a test suite composed of 5 videos randomly picked among our 49 videos. We ran the 12 selected programs on this randomly picked test suite. For each program, similarly as presented in Figure 7, we computed the Precision measure averaged over the test suite. Then, we confront the classification of morphs (depending on whether the average of their performance is above 0.5 or below 0.4 ) with the intuition of the expert and count how many times they disagree. To mitigate the potential bias induced by random picking, we run this process 10 times. The result is that a minimum of 2 programs out of 12 have been misclassified, with a worst case of 5 misclassified programs.

In average, over the 10 runs, almost 4 programs over 12 are misclassified. The associated standard deviation is 1 , suggesting that overall, between 3 and 5 programs are likely to be misclassified. As a conclusion, we got some evidence that our optimal test suite of 5 videos performs significantly better that a random one of the same size to tell good from poor configurations apart.

\subsection{2 (COCO case) Can a smaller test suite built such that it maximizes its dispersion score provide a similar ranking of morphs as the original test suite?}

Again, we created a reduced test suite containing

5 concepts following the same process as before. The 5 concepts are presented in Table 4 and yield a score of 0.673 . It is worth noting that it is very close to the dispersion score of the entire COCO benchmark. Note that, once again, the choice of these 5 concepts are not the same as picking the 5 top rows of Table 5 in Appendix. We have also stated in Section 3.3.1 that "Sports" and "Electronic" yielded the highest dispersion scores. "Electronic" has been chosen in our minimized test suite but using "Sports" would have been the same as long as it would have activated the same bins.

With this new smaller benchmark, we rank again the competitors. We check that the two rankings are similar in order to assess that not all categories are needed when performing continuous evaluations of morphs' performances. The similarity between the two rankings is established using the Spearman correlation coefficient. This coefficient indicates whether two ranked lists are strongly correlated when the value is close to 1 or -1 , showing whether the evolution follows the same tendency or opposite directions. On the other hand, if the coefficient is close to 0 , then no correlation can be deduced.

Table 7 in Appendix provides a comparison of performances of a sample of competitors' techniques w.r.t. the two benchmarks. The first column presents competitors' names. We selected 13 techniques out of the 52 competitors that are shown on the leaderboard. That is, we show approximately one out of five techniques. The second column shows the performances provided by the leaderboard. We assume such performances to be an average over all 12 concepts.

The third column shows the averaged performances over the set of 5 concepts (see Table 4) that we retrieved from our method. Finally, the last column shows the absolute difference between the two performances. The differences are rather low which shows how close we are from the original measures but considering fewer data.

We also computed some statistics over all 52 techniques which compare the differences between the two set of performances. The average difference in performances over all 52 techniques is $\approx 0.013$ with a standard deviation of $\approx 0.004$. This shows that, most of the time, differences in retrieved performances are in the range $[0.01 ; 0.02]$ approximately. We also searched for the maximum and minimum differences, they are respectively $\approx 0.025$ and 0.008 .

Regarding ranks directly: Table 8, in Appendix, shows two rankings on the second and third columns. First, the one retrieved from the COCO leaderboard. Second, the one we computed considering our reduced benchmark. The two rankings are similar with only a few ranks that are permuted with the one above or below. The result Spearman correlation coefficient of 0.998 shows a strong correlation between the two rankings.

This strong correlation shows that a small subset selected through to the notion of the dispersion score is nearly as powerfull as the full test suite to rank competitors. The concrete consequence of that is out of all concepts in COCO, only a smaller number is needed to assess the global behavior of competitors. In the end, their continuous evaluations could be reduced to assess performances on a smaller number of concepts such that competitors can get an idea of their rank in the competition more quickly, with fewer resources which in turn could help them improve their solutions more quickly and more frequently. 
3.4.3 (Haxe case) Can we discover bugs thanks to the dispersion score? Can we build a smaller test suite that is able to select interesting test cases?

For this case, we want to check that a higher dispersion score might be correlated with the detection of performance bugs. In particular, we focus on the test suite with a maximal dispersion score that had activated 3 bins.

Because most dispersion scores activated one or two bins, we assumed that performances observed lied close to a boundary between those two bins and little variations caused observations to lie sometimes in one bin and sometimes in another. However, when three bins are activated, it is unlikely that little variations could cause this behavior. There must be something else: we analyzed observations of the test suite associated with the highest retrieved dispersion score. Retrieved performance were rather consistent except for code generator variants targeting the PHP language. Performances regarding those morphs drastically increased. In fact, execution times were at least 40 times longer than for morphs targeting other languages. Checking results with authors from previous work [16], [17], they also have noticed this anomaly and reported it to Haxe community in a bug report. Developers responded that they knew about it, it was fixed already but the patch was not live when [16], [17] conducted their study.

\subsection{Concluding remarks over the method}

We can answer the main research questions.

Performance diversity (RQ1). Is our dispersion score able to capture different performance values and thus assign different scores to different test suites? We showed we are able to build a dispersion score that assigns a higher value to test suites able to capture significant differences in performance values. The ranges of dispersion scores are as follows: the OpenCV case showed dispersion scores ranging from 0.08 to 0.207 . The minimal score of 0.08 is assigned to a test suite on which most of OpenCV morphs performed similarly. On the other hand, with a score of 0.207 , about a fifth of morphs performed significantly different on this test suite. Regarding COCO and Haxe, dispersion scores ranged respectively from 0.308 to 0.423 and from 0.047 to 0.143 . For every case studies, dispersion scores were able to differentiate test cases by assigning them different dispersion score whether they are able to capture more or less different performance values.

Stability (RQ1). Are dispersion scores very sensitive to the use of particular morphs? Our sensitivity analysis showed that dispersion scores were rather stable regardless of used morphs. Hence our method is able to deal with morphs having similar performances; we can certainly avoid the costly use of some equivalent morphs. It also suggests that our method is robust even when the selection of morphs is realized in an agnostic way being the use of random selection. However, we cannot claim that domain knowledge will not be beneficial to our method (e.g., for selecting optimal configurations and morphs, see discussions in Section 4).

Applicability (RQ2). Can dispersion scores be correlated with an external evaluation, specific to each case, of what would be "good" test suites? We showed that, dispersion scores can be used to rank test suites in order of importance w.r.t. the different performance values they are able to capture. They can also be used to build smaller test suites than the original that will maximize this score. With smaller test suites, we were able to:

- execute a set of OpenCV morphs selected by a Computer Vision expert and match the intuition of the experts regarding morphs' behaviors (i.e., the 6 morphs supposed to perform better according to the expert were indeed the ones performing the best on our smaller test suite composed of 5 test cases);

- retrieve a similar ranking of COCO competitors as the original one, that is available online, with a strong Spearman correlation coefficient (i.e., 0.998) between the two rankings. Our test suite took into account 5 of the 12 concepts originally present in the COCO dataset;

- exhibit a bug in generated PHP code with only 5 test suites out of the 84 composing the original test suite. Of course, this bug was already found by the original test suite. From previous studies [16], [17], this bug was the only one to be found via a comparison of performances. By applying Multimorphic Testing, we were able to reduce the size of the test suite while keeping its ability to show this performance bug.

\subsection{Reproducibility of experiments}

Data, code and results are available in a public repository on Github at the following link: https: //github.com/templep/TSE_MM_test.git.

For practitioners interested in reproducing the analysis of our data, we provide all configurations, test suites and observations through CSV files. Statistical results presented this article are available through text files or plots. The code for producing such results is written in Scilab, an open-source software close to Matlab and is also included. For OpenCV and Haxe practitioners interested in reproducing the computations of performance data, we provide specific instruc- 
tions as well as the code to instrument the whole process.

\section{Discussions and Threats to Va- LIDITY}

\subsection{Internal threats}

To compute our dispersion score, we used a 2D representation inspired from histograms. Despite not being interested in the actual frequency value of each bin, we need to know which one are activated to compute our dispersion score. On the $\mathrm{X}$-axis, the representation defines bins and their number is crucial in our method. Defining the right number of bins remains an open question since it depends on the application: trying to analyze a color distribution of an image, comparing two different data distribution requires a very fine-grained analysis and thus a larger number of bins; while, in our case, requirements are different. Using a small number of bins would provide a coarse analysis of the variability in the results while more bins might isolate every execution into its own single bin and thus would show differences that are not significant. We fixed the number of bins to the number of used morphs as a reasonable trade-off, but this is only true if the number of morphs is large enough.

In RQ2, we validate the usefulness of our dispersion score by building test suites composed of 5 test cases that maximized the dispersion scores. 5 is an arbitrary value chosen to limit the amount of time taken by the exhaustive search. For example, in the OpenCV case, 5 test suites among 49 result in $49 ! /(5 ! *(49-5) !)$ combinations which is about $1.9 \mathrm{M}$ combinations. Increasing the number of test suites that we take into account drastically increases the number of combinations, for instance, taking 7 instead of 5 results in about $85.9 \mathrm{M}$ combinations. We are aware that, depending on the application domain, the number of test cases to consider may vary and 5 is certainly not the optimal number to use in every occasion. 5 has no value per se in our experiments except that it allows us to make computation time affordable for our validation. Even with such a small number, we already get interesting enough results to be able to conclude on the potential of our technique.

In the sensitivity analysis, we analysed the effect of removing half of the morphs. This choice is arbitrary but seems reasonable to demonstrate the stability of the dispersion score and compare it with the use of all morphs. It is an open question how many morphs should be chosen to obtain a relevant score.

In the end, our dispersion score, the representation and exhaustive search are only one way to instantiate the Multimorphic testing approach. Even if it seems to work surprisingly well, at least for the different application we have considered, other options might perform better and need to be explored.

\subsection{External threats}

Applicability. Can Multimorphic testing and our proposed dispersion score be used in different domains? Our experiments took three different application domains that are tracking of objects in videos, object recognition in images and program generation. The method was able to detect test suites emphasizing interesting behaviors of morphs. While two application domains are rather close, associated tasks were different. Results presented in Section 3 mitigate this first threat as it shows that, at least in presented situations, Multimorphic testing can be applied.

Performance dimensions and metrics. About generalization, we presented results regarding only one performance measure at a time, namely Precision or execution time. Further experiments have been running taking into account other measures such as Recall or memory consumption or even a combination of Precision and Recall or Precision and execution time, similar conclusions were drawn from those extra experiments but we do not show them in this document as it would not provide more insights about the method. They are available on the companion GitHub repository though.

On test suites (OpenCV). Regarding the OpenCV case specifically, test sets were composed of synthetic videos only. The merit of synthetic videos is that (1) the associated ground truths are of high quality (by construction since they are synthesized); (2) we can better control the properties of the videos and thus increase the diversity of situations. Synthetic videos are getting more and more used in the industry or in research as a substitute or complement of real assets [9], [18], [19], [20]. However, natural videos may not provide as diverse behaviors and performance measurements as we observed. Note that other experiments like the COCO case used "natural" images and still gave fairly good results which mitigates this threat.

On test suites (Coco). Focusing on the $\mathrm{COCO}$ dataset, we did not have access to raw images; we only considered concepts and classes of objects. It seems realistic to assume that object classes are not equally represented over all the images of the dataset. For instance, there might be fewer objects labeled "hair dryer" than objects labeled "cat". A hypothesis is that classes that are more represented might have more chances to provide "extra diversity" and thus to show differences among competing approaches. Results we present in Section 3 might be biased as they could simply reveal that the reduced set of 5 concepts is composed of the 5 most represented ones. 
However, it is only a hypothesis that does not necessarily hold in general, i.e., there is not necessarily a correlation between the size of the dataset and performance diversity. For COCO, we can hardly validate this hypothesis since concepts are coarse abstractions averaging performances of large subsets of images. In any case, we have shown that our method is able to retain the key elements of the dataset that exhibit diversity within the performances of competing systems.

Data preprocessing. In the Haxe case, our evaluation is based on results from prior measures performed on the same Haxe code generator. Measures that we retrieved were preprocessed (as explained in 3.2.3) which might exacerbate or alleviate differences in observed performances. However, the fact that we were able to retrieve a test suite that highlighted the presence of a bug in PHP generated code is encouraging. Furthermore, the fact that measures were consistent, with only one or two bins activated, shows that the dispersion score is somehow invariant to this preprocessing. Further investigations should be conducted in order to understand which kind of preprocessings do not affect drastically the dispersion score.

Morphs' selection The ability of Multimorphic Testing to observe different performance behavior is dependent of the nature of morphs. Using only very similar morphs (with only a small delta in the value of one parameter) might not be enough to observe differences while the morphs will be different in their configurations. Used morphs should be somehow representative of the whole population of morphs of a system. This is an assumption that we used for all our experiments: we have generated morphs of OpenCV for a specific task with the goal of exploring the configuration space as much as possible; we considered competitors to the COCO competitions to be representative of the state-ofthe-art techniques in terms of object recognition; and similarly for the Haxe case, we considered that target languages and optimization options to be representative of what users might look for.

The underlying problem is how to sample those morphs efficiently? This remains an open problem in the Software Product Lines community that is addressed by several works, mainly for finding functional bugs [21], [22], [23], [24]. Overall, a threat to the adoption of multimorphic testing is that the process can be highly computational demanding for some domains; an optimal selection of morphs and reduction of performance test suites are open research directions worth exploring.

\section{Related Work}

This paper proposes to proactively create morphs, also called variants in the Software
Product Lines community, for the purpose of assessing the quality of a test suite. We have evaluated our approach in different application domains. Our contribution is thus at the crossroad of software testing (including mutation and metamorphic testing ${ }^{10}$ ), variability management, compilers, and computer vision.

\subsection{Software testing}

Software testing techniques have been developed to assess, optimize, or generate a test suite. The general idea is that a test suite could be evaluated according to a certain criterion, e.g., a code coverage criterion describing a set of structural aspects of a program, like statements, lines or branches. Search-based techniques are particularly used to explore this search space to find the input data that maximize the given objective [25] (e.g., cover as many branches as possible [26]). Black-box or white-box software testing techniques have been proposed [27]. The idea is to generate random inputs while observing resulting outputs, typically for detecting failures. Whitebox fuzzing, such as SAGE, consists of executing the program and gathering constraints on inputs [28]. SAGE exploits constraints to guide the test generation.

While load testing techniques have been developed to evaluate the performance of systems [29], [30], no such testing criteria exist for quantitative properties. We developed a dedicated process and criterion that can be defined in terms of performance dispersion of morphs. Based on such a criterion, we can envision to optimize test suites using, for instance, searchbased techniques.

Mutation analysis is a well-known technique for either evaluating test suite quality or supporting test generation [31], [32], [33], [25]. Artificial defects, known as mutations, are injected into the program yielding mutants; one can then measure test effectiveness based on the number of "killed" mutants that have failed tests. Multimorphic testing pursues similar goal as mutation testing, i.e., assessment of the strength of a test suite, with our morphs playing a similar role as mutants do in mutation analysis. There are two important differences. First, we do not rely on typical mutation operators but on parametrization (see Section 2) to generate our variants. Parametrization is much more simple to instrument using offthe-shelf product line technology: we have more control and limit the risk of having unrealistic variants. Second, and more importantly, instead

10. Actually some people say that what we are doing here is mutation testing, while others say equally strongly that it is metamorphic testing, and still others say it is neither. We thus forged a new term, Multimorphic testing, but if there is a consensus among the reviewers that this is either mutation testing or metamorphic testing, we would be happy to acknowledge and change our title. 
of giving pass/fail verdicts, our tests yield quantitative values for properties such as execution time, precision or recall. Looking at these raw numerical values, we cannot conclude whether a variant is "killed" by a given test. We thus developed a dispersion score for quantifying the ability of a test suite to reveal differences in such quantitative properties.

Segura et al. [34] surveyed metamorphic testing. It can be used to compare different programs for the same input and determine whether some relations are kept from one execution to another [35]. Recently, some works discuss the use of metamorphic testing [17], [36] to alleviate the test oracle problem in the context of non functional properties. Applied to our domain, the idea is to specify from a large set of test cases, in our case being input programs, the relation between these inputs and the expected qualitative properties outputs. In our case, such properties are the expected performance of the output programs in terms of CPU and memory. However, these works do not aim to evaluate the quality of a test suite.

To conclude and to the best of our knowledge, only a few works consider quantitative properties or performances for the quality measure of a test suite despite a vast literature on mutation and metamorphic testing.

\subsection{Testing and variability}

Numerous techniques have been developed to efficiently verify a family of programs, a.k.a. software product lines or configurable systems, based on testing, type checking, model checking, or theorem proving [37].

For instance, prior work has considered the problems of optimizing the execution of tests for a set of related products [38], [39], [40], sampling the configurations to test [41], [42], [43], [44], [9], [24], [45], [46], or identifying relevant products to test [37], [47]. In this paper, we do not aim to verify an existing product line; we synthetically create a family of variants with the objective of assessing the quality of a test suite. An open research direction is to investigate how variability techniques can benefit to our Multimorphic testing framework. In particular, the handling of quantitative properties and the nature of test data, such as large videos, input programs, challenges existing techniques. Incidentally, our testing method could benefit to software product line engineering, either for assessing a test suite or for exploring the performance of different variants (see e.g., [48], [49]).

\subsection{Testing and code generators}

Most of the previous work on code generator testing focuses on checking the correct functional behavior of generated code [50], [51]. Most of these research efforts rely on the comparison of the model execution to the generated code execution. This is known in the software testing community as equivalence, comparative, or backto-back testing approach [52], [53].

For instance, Stuermer et al. [50] present a systematic test approach for model-based code generators. They investigate the impact of optimization rules for model-based code generation by comparing the output of the code execution with the output of the model execution. If these outputs are equivalent, it is assumed that the code generator works as expected. They evaluate the effectiveness of their approach by means of optimizations performed by the TargetLink code generator. They use Simulink as a simulation environment of models. In [54], authors present a testing approach of the Genesys code generator framework which tests the translation performed by a code generator from a semantic perspective rather that just checking for syntactic correctness of the generation result. Basically, Genesys realizes back-to-back testing by executing both the source model as well as the generated code on top of different target platforms. Both executions produce traces and execution footprints which are then compared. The limitation of these testing approaches is that they are applicable only when the input model/source code is executable. Compared to our proposal, we rather propose an approach that detects "bad" code generator configurations at the source code level regardless of the input model/source code execution.

Previous work on non-functional testing of code generators focuses on comparing, as oracle, the non-functional properties of hand-written code to automatically generated code [55], [56]. As an example, Strekelj et al. [15] implemented a simple 2D game in both, the Haxe programming language and the target programming language, and evaluated the difference in performance between the two versions of code. They showed that the generated code through Haxe has better performance than the hand-written one.

\subsection{Testing and computer vision}

Numerous benchmarks or test suites are created for evaluating and comparing computer vision systems [57], [12], [14], [13], [58], [59] and similar initiatives are observed in other application domains like natural language processing, satisfiability solvers. This brings up questions about the quality of benchmarks.

For instance, Ponce et al. [60] analyze existing image classification test datasets and report several biases including orientations and lack of clutter. CV-HAZOP [61] proposes to use a checklist that would help testers understand which visual difficulties such as changes in illumination 
or partial occlusion are more represented in a benchmark. Such checklists can help ensure that at least a representative of a difficulty is present in the final benchmark but it does not tell anything about how well techniques are able to deal with it. Multimorphic testing proposes a method to actually measure this.

Some testing techniques assume that, to be efficient, tests should pass through every line of code of a program. Underlying assumptions are that if all lines of code are covered then the program will not break at the first execution. Recently DeepXplore [62] proposed a related metric for Deep Learning algorithms, stating that if test suites are able to pass through every neuron of a Neural Network then test ensures that the Neural Network will not break down at the first execution. While DeepXplore can give an idea of the stability of produced code and help to detect bugs, it does not allow to know which aspects (e.g., which kind of inputs) should be taken into account. DeepTest [63] uses neuron coverage for guided test generation and leverage metamorphic relations to identify erroneous behaviors in a single neural network. The goal of multimorphic testing is to characterize the quality of existing test suites, not to generate new test cases. DeepXplore and DeepTest are whitebox or grey-box testing techniques and assume to have access to internal details of a Neural Network. In contrast, Multimorphic testing is a black-box approach that can be applied to any kind of program provided it features some kind of internal variability.

\section{Conclusion}

We applied multimorphic testing to assess the effectiveness of a test suite in revealing performance weaknesses of different systems. We showed that our method can be applied for quantitative properties such as precision, recall or execution time. The core idea was to generate system variants, that we called morphs, by varying their parameters' values and to check whether it makes any difference on the outcome of tests in terms of such quantitative properties. Intuitively a "good" test has a good discriminating power over the set of morphs. Conversely, a "bad" test features more or less the same results whatever the morphs and thus should be considered as useless from a performance testing point of view. We have proposed to use the dispersion score to embody this intuition, and have empirically shown over 3 different applications its applicability. We have also shown its usefulness regarding different goals that are detailed for every application. Above all, thanks to our method, we can envision to remove unnecessary, redundant test cases from test suites, or improve existing test data sets.
Applications of Multimorphic testing. All our evaluations have been conducted with respect to the objective of minimizing existing test suites. However, we envision other applications to Multimorphic testing. For instance, the score could help in prioritizing the execution of test cases, typically in the context of continuous integration. Tests with higher dispersion scores should be executed first, until the time budget allocated for testing is expanded. We can also think about Metamorphic Testing as a test criterion similarly to the use of code coverage - of course the same caveat applies. As code coverage or more generally structural testing is a way to quantify how much of the code has been executed by the test suite, we could consider Multimorphic testing as a way to quantify how much performances may differ using a specific test suite. Once this quantity is known, it can become a criterion on its own to evaluate the quality of a test suite for a given application domain, and practitioners may express requirements about a certain level of dispersion to reach before the test suite is considered of "sufficient" quality. This application is directly related to another one which would be test suite improvement. Being able to measure the dispersion reached by a test suite can indeed help ringing a bell in the head of testers, for example when the score is really low. Multimorphic testing can encourage developers to find new test cases that increase the dispersion of the test suite and hopefully create new "unusual", not considered before corner cases. Another obvious research direction would be to combine Multimorphic testing to well-known test selection techniques such as search-based techniques in order to concretely generate optimal test suites, thereby providing cheaper and better test suites than current hand-crafted benchmarks. Finally, we can consider Multimorphic testing as a first step towards test suite and program understanding. Being able to use test cases that show different behaviors from the morphs or program variants may help developers think about why some other techniques work better that theirs on a specific input. Once the question is raised, they can further improve their technique in order to reach the same level of performance as the targeted one. Developers can review suboptimal morphs and potentially identify performance weaknesses of some configurations.

Future work includes investigating other dispersion metrics, since we do not claim the one proposed in this article is the best possible one. Also, we would like to pursue the idea of using this method in different contexts. We could use different code generators and compilers (e.g., ThingML, Num, TypeScript) but also try investigate new domains like databases. Other candidate domains are highly configurable systems 
featuring some form of recognition (e.g., video or speech recognition) or more generally any software applications where quantitative properties are of prior importance.

\section{Acknowledgment}

We would like to thank Bruno Sericola from Inria Rennes-Bretagne Atlantique who helped us in the formalization of our work. This research was partially funded by the ANR-17-CE25-001001 VaryVary project.

\section{REFERENCES}

[1] P. Temple, M. Acher, and J.-M. Jézéquel, "Poster: Multimorphic Testing," in ACM/IEEE 40th International Conference on Software Engineering: Companion Proceedings, Gothenburg, Sweden, May 2018, pp. 1-2. [Online]. Available: https://hal.inria.fr/hal-01730163

[2] K. Bak, K. Czarnecki, and A. Wasowski, "Feature and meta-models in clafer: mixed, specialized, and coupled," in SLE'10, 2011.

[3] S. Apel, D. Batory, C. Kästner, and G. Saake, FeatureOriented Software Product Lines: Concepts and Implementation. Springer-Verlag, 2013.

[4] B. W. Silverman, Density estimation for statistics and data analysis. Routledge, 2018.

[5] D. W. Scott, "On optimal and data-based histograms," Biometrika, vol. 66, no. 3, pp. 605-610, 1979.

[6] - Multivariate density estimation: theory, practice, and visualization. John Wiley \& Sons, 2015.

[7] M. Acher, P. Collet, P. Lahire, and R. France, "Familiar: A domain-specific language for large scale management of feature models," Science of Computer Programming (SCP), vol. 78, no. 6, pp. 657-681, 2013.

[8] T. Thüm, C. Kstner, F. Benduhn, J. Meinicke, G. Saake, and T. Leich, "Featureide: An extensible framework for feature-oriented software development," Science of Computer Programming, 2012.

[9] J. A. Galindo, M. Alférez, M. Acher, B. Baudry, and D. Benavides, "A variability-based testing approach for synthesizing video sequences," in International Symposium on Software Testing and Analysis, ser. ISSTA 2014. ACM, 2014, pp. 293-303. [Online]. Available: http://doi.acm.org/10.1145/2610384.2610411

[10] M. Alférez, M. Acher, J. A. Galindo, B. Baudry, and D. Benavides, "Modeling Variability in the Video Domain: Language and Experience Report," Software Quality Journal, pp. 1-28, Jan. 2018. [Online]. Available: https://hal.inria.fr/hal-01688247

[11] J. Deng, W. Dong, R. Socher, L.-J. Li, K. Li, and L. Fei-Fei, "Imagenet: A large-scale hierarchical image database," in Computer Vision and Pattern Recognition, 2009. CVPR 2009. IEEE Conference on. IEEE 2009, pp. 248-255.

[12] T. Lin, M. Maire, S. J. Belongie, L. D. Bourdev, R. B. Girshick, J. Hays, P. Perona, D. Ramanan, P. Dollár, and C. L. Zitnick, "Microsoft COCO: common objects in context," CoRR, vol. abs/1405.0312, 2014. [Online] Available: http://arxiv.org/abs/1405.0312

[13] "Pets 2016 dataset," http:// www.cvg.reading.ac.uk/P

[14] G. Griffin, A. Holub, and P. Perona, "Caltech256 image dataset," 2006. [Online]. Available: http://www. vision.caltech.edu/Image_Datasets/Caltech256/

[15] D. Štrekelj, H. Leventić, and I. Galić, "Performance overhead of haxe programming language for crossplatform game development," International Journal of Electrical and Computer Engineering Systems, vol. 6, no. 1, pp. 9-13, 2015.

[16] M. Boussaa, O. Barais, B. Baudry, and G. Sunyé, "Automatic non-functional testing of code generators families," in Proceedings of the 2016 ACM SIGPLAN International Conference on Generative Programming: Concepts and Experiences. ACM, 2016, pp. 202-212.
[17] M. Boussaa, "Automatic Non-functional Testing and Tuning of Configurable Generators," Ph.D. dissertation, Inria Rennes - Bretagne Atlantique ; University of Rennes 1, 2017. [Online]. Available: https://hal.archives-ouvertes.fr/tel-01598821

[18] M. Johnson-Roberson, C. Barto, R. Mehta, S. N. Sridhar, and R. Vasudevan, "Driving in the matrix: Can virtual worlds replace human-generated annotations for real world tasks?" CoRR, vol. abs/1610.01983, 2016. [Online]. Available: http: //arxiv.org/abs/1610.01983

[19] M. Patrick, M. D. Castle, R. O. J. H. Stutt, and C. A. Gilligan, "Automatic test image generation using procedural noise," in Proceedings of the 31st IEEE/ACM International Conference on Automated Software Engineering, ASE 2016, Singapore, September 3-7, 2016, 2016, pp. 654-659. [Online]. Available: http://doi.acm.org/10.1145/2970276.2970333

[20] A. Shrivastava, T. Pfister, O. Tuzel, J. Susskind, W. Wang, and R. Webb, "Learning from simulated and unsupervised images through adversarial training," arXiv preprint arXiv:1612.07828, 2016.

[21] J. Oh, D. S. Batory, M. Myers, and N. Siegmund, "Finding near-optimal configurations in product lines by random sampling," in Proceedings of the 2017 11th Joint Meeting on Foundations of Software Engineering, ESEC/FSE 2017, Paderborn, Germany, September 4-8, 2017, 2017, pp. 61-71. [Online]. Available: http://doi.acm.org/10.1145/3106237.3106273

[22] K. Czarnecki, S. She, and A. Wasowski, "Sample spaces and feature models: There and back again," in SPLC, 2008.

[23] A. Sarkar, J. Guo, N. Siegmund, S. Apel, and K. Czarnecki, "Cost-efficient sampling for performance prediction of configurable systems (t)," in $A S E^{\prime} 15,2015$.

[24] F. Medeiros, C. Kästner, M. Ribeiro, R. Gheyi, and S. Apel, "A comparison of 10 sampling algorithms for configurable systems," in ICSE'16, 2016.

[25] J. M. Rojas, M. Vivanti, A. Arcuri, and G. Fraser, "A detailed investigation of the effectiveness of whole test suite generation," Empirical Software Engineering, vol. 22, no. 2, pp. 852-893, 2017. [Online]. Available: http://dx.doi.org/10.1007/s10664-015-9424-2

[26] J. C. Miller and C. J. Maloney, "Systematic mistake analysis of digital computer programs," Commun. ACM, vol. 6, no. 2, pp. 58-63, Feb. 1963. [Online]. Available: http://doi.acm.org/10. $1145 / 366246.366248$

[27] P. Godefroid, N. Klarlund, and K. Sen, "Dart: Directed automated random testing," SIGPLAN Not., 2005. [Online]. Available: http://doi.acm.org/10. 1145/1064978.1065036

[28] P. Godefroid, M. Y. Levin, and D. Molnar, "Sage: Whitebox fuzzing for security testing," Queue, 2012. [Online]. Available: http://doi.acm.org/10. $1145 / 2090147.2094081$

[29] H. Malik, H. Hemmati, and A. E. Hassan, "Automatic detection of performance deviations in the load testing of large scale systems," in Proceedings of the 2013 International Conference on Software Engineering, ser. ICSE '13. Piscataway, NJ, USA: IEEE Press, 2013, pp. 1012-1021. [Online]. Available: http:/ / dl.acm.org/citation.cfm?id=2486788.2486927

[0]16/.. M. Jiang, "Automated analysis of load testing results," in Proceedings of the 19th International Symposium on Software Testing and Analysis, ser. ISSTA '10. New York, NY, USA: ACM, 2010, pp. 143-146. [Online]. Available: http://doi.acm.org/10. $1145 / 1831708.1831726$

[31] J. H. Andrews, L. C. Briand, Y. Labiche, and A. S. Namin, "Using mutation analysis for assessing and comparing testing coverage criteria," IEEE Transactions on Software Engineering, vol. 32, no. 8, pp. 608624, Aug 2006.

[32] M. Gligoric, A. Groce, C. Zhang, R. Sharma, M. A. Alipour, and D. Marinov, "Comparing non-adequate test suites using coverage criteria," in Proceedings of 
the 2013 International Symposium on Software Testing and Analysis, ser. ISSTA 2013. New York, NY, USA: ACM, 2013, pp. 302-313. [Online]. Available: http://doi.acm.org/10.1145/2483760.2483769

[33] M. Papadakis and N. Malevris, "Automatic mutation test case generation via dynamic symbolic execution," in 2010 IEEE 21st International Symposium on Software Reliability Engineering, Nov 2010, pp. 121130.

[34] S. Segura, G. Fraser, A. B. Sánchez, and A. R. Cortés, "A survey on metamorphic testing," IEEE Trans. Software Eng., vol. 42, no. 9, pp. 805-824, 2016. [Online]. Available: http://dx.doi.org/10.1109/TSE. 2016.2532875

[35] E. T. Barr, M. Harman, P. McMinn, M. Shahbaz, and S. Yoo, "The oracle problem in software testing: A survey," IEEE Transactions on Software Engineering, vol. 41, no. 5, pp. 507-525, May 2015.

[36] S. Segura, J. Troya, A. D. Toro, and A. R. Cortés, "Performance metamorphic testing: Motivation and challenges," in 39th IEEE/ACM International Conference on Software Engineering: New Ideas and Emerging Technologies Results Track, ICSE-NIER 2017, Buenos Aires, Argentina, May 20-28, 2017, 2017, pp. 7-10.

[37] T. Thüm, S. Apel, C. Kästner, I. Schaefer, and G. Saake, "A classification and survey of analysis strategies for software product lines," ACM Computing Surveys, 2014.

[38] C. Kim, S. Khurshid, and D. Batory, "Shared execution for efficiently testing product lines," in Software Reliability Engineering (ISSRE), 2012, 2012.

[39] H. V. Nguyen, C. Kästner, and T. N. Nguyen, “Exploring variability-aware execution for testing pluginbased web applications," in ICSE'14, 2014.

[40] X. Devroey, G. Perrouin, M. Papadakis, A. Legay, P.-Y. Schobbens, and P. Heymans, "Featured modelbased mutation analysis," in Proceedings of the 38th International Conference on Software Engineering, ser ICSE '16. New York, NY, USA: ACM, 2016, pp. 655-666. [Online]. Available: http://doi.acm.org/10. $1145 / 2884781.2884821$

[41] M. F. Johansen, Ø. Haugen, and F. Fleurey, "An algorithm for generating $\mathrm{t}$-wise covering arrays from large feature models," in 16th International Software Product Line Conference, SPLC '12, 2012, pp. 46-55.

[42] C. Henard, M. Papadakis, G. Perrouin, J. Klein, P. Heymans, and Y. L. Traon, "Bypassing the combinatorial explosion: Using similarity to generate and prioritize t-wise test configurations for software product lines," IEEE Trans. Software Eng., 2014.

[43] B. Pérez Lamancha and M. Polo Usaola, "Testing Product Generation in Software Product Lines Using Pairwise for Features Coverage," in 22nd IFIP WG 6.1 International Conference on Testing Software and Systems (ICTSS), ser. Testing Software and Systems, A. P. A S. J. C. Maldonado, Ed., vol. LNCS-6435. Natal, Brazil: Springer, Nov. 2010, pp. 111-125. [Online]. Available: https:/ / hal.inria.fr/hal-01055240

[44] M. B. Cohen, M. B. Dwyer, and J. Shi, "Constructing interaction test suites for highly-configurable systems in the presence of constraints: A greedy approach," IEEE Transactions on Software Engineering, vol. 34, no. 5, pp. 633-650, Sep. 2008.

[45] A. Halin, A. Nuttinck, M. Acher, X. Devroey, G. Perrouin, and B. Baudry, "Test them all, is it worth it? assessing configuration sampling on the jhipster web development stack," Empirical Software Engineering, vol. 24, no. 2, pp. 674-717, 2019. [Online]. Available: https://doi.org/10.1007/ s10664-018-9635-4

[46] C. Yilmaz, M. B. Cohen, and A. A. Porter, "Covering arrays for efficient fault characterization in complex configuration spaces," IEEE Transactions on Software Engineering, vol. 32, no. 1, pp. 20-34, 2006.

[47] C. H. P. Kim, D. Marinov, S. Khurshid, D. Batory, S. Souto, P. Barros, and M. D.Amorim, "Splat: Lightweight dynamic analysis for reducing combina- torics in testing configurable systems," in ESEC/FSE 2013, 2013.

[48] J. Guo, E. Zulkoski, R. Olaechea, D. Rayside, K. Czarnecki, S. Apel, and J. M. Atlee, "Scaling exact multiobjective combinatorial optimization by parallelization," in ASE. ACM, 2014.

[49] C. Henard, M. Papadakis, M. Harman, and Y. L. Traon, "Combining multi-objective search and constraint solving for configuring large software product lines," in 37th IEEE/ACM International Conference on Software Engineering, ICSE 2015, Florence, Italy, May 16-24, 2015, Volume 1, 2015, pp. 517-528. [Online]. Available: http://dx.doi.org/10. 1109/ICSE.2015.69

[50] I. Stuermer, M. Conrad, H. Doerr, and P. Pepper, "Systematic testing of model-based code generators," IEEE Transactions on Software Engineering, vol. 33, no. 9, p. 622, 2007.

[51] I. Sturmer and M. Conrad, "Test suite design for code generation tools," in Automated Software Engineering, 2003. Proceedings. 18th IEEE International Conference on. IEEE, 2003, pp. 286-290.

[52] W. M. McKeeman, "Differential testing for software," Digital Technical Journal, vol. 10, no. 1, pp. 100-107, 1998.

[53] M. A. Vouk, "Back-to-back testing," Information and software technology, vol. 32, no. 1, pp. 34-45, 1990.

[54] S. Jörges and B. Steffen, "Back-to-back testing of model-based code generators," in International Symposium On Leveraging Applications of Formal Methods, Verification and Validation. Springer, 2014, pp. 425444.

[55] S. Stepasyuk and Y. Paunov, "Evaluating the haxe programming language-performance comparison between haxe and platform-specific languages," Ph.D. dissertation, University of Gothenburg, 2015.

[56] J. Richard-Foy, O. Barais, and J.-M. Jézéquel, "Efficient high-level abstractions for web programming," in ACM SIGPLAN Notices, vol. 49, no. 3. ACM, 2013, pp. 53-60.

[57] M. Everingham, S. M. A. Eslami, L. Van Gool, C. K. I. Williams, J. Winn, and A. Zisserman, "The pascal visual object classes challenge: A retrospective," International Journal of Computer Vision, vol. 111, no. 1, pp. 98-136, Jan. 2015

[58] A.-T. Nghiem, F. Bremond, M. Thonnat, and M. Ruihua, "A New Evaluation Approach for Video Processing Algorithms," in IEEE Workshop on Motion and Video Computing, Austin, Texas, United States, Feb. 2007. [Online]. Available: https: / / hal.inria.fr/inria-00502955

[59] A. T. Nghiem, F. Bremond, M. Thonnat, and V. Valentin, "Etiseo, performance evaluation for video surveillance systems," in 2007 IEEE Conference on Advanced Video and Signal Based Surveillance, Sept 2007, pp. 476-481.

[60] J. Ponce, T. Berg, M. Everingham, D. Forsyth, M. Hebert, S. Lazebnik, M. Marszalek, C. Schmid, B. Russell, A. Torralba, C. Williams, J. Zhang, and A. Zisserman, "Dataset issues in object recognition," in Towards Category-Level Object Recognition, ser. Lecture Notes in Computer Science (LNCS), J. Ponce, M. Hebert, C. Schmid, and A. Zisserman, Eds. Springer, 2006, vol. 4170, pp. 29-48. [Online]. Available: https://hal.inria.fr/inria-00548595

[61] O. Zendel, M. Murschitz, M. Humenberger, and W. Herzner, "Cv-hazop: Introducing test data validation for computer vision," in 2015 IEEE International Conference on Computer Vision (ICCV), Dec 2015, pp. 2066-2074.

[62] K. Pei, Y. Cao, J. Yang, and S. Jana, “Deepxplore: Automated whitebox testing of deep learning systems," CoRR, vol. abs/1705.06640, 2017. [Online]. Available: http:/ / arxiv.org/abs/1705.06640

[63] Y. Tian, K. Pei, S. Jana, and B. Ray, "Deeptest: Automated testing of deep-neural-network-driven 
autonomous cars," CoRR, vol. abs/1708.08559, 2017. [Online]. Available: http://arxiv.org/abs/1708.08559

\section{AUthoR's BIOGRAPHIES}

Paul Temple is a $\mathrm{PhD}$ student in the DiverSE team at the University of Rennes 1 since September 2015. He received a Master's degree in Computer Science from the University of Rennes 1 in 2013 and went in the e-

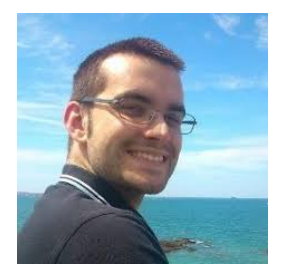
payments and biometrics team (part of ENSICaen in Caen, FRANCE) during one year starting July 2014 as an engineer. He is interested in Data and Image Processing, Software Engineering and Machine Learning. He is now working on how to leverage machine learning techniques to ease the selection of a product derived from configurable systems w.r.t. users' requirements.

Dr. Mathieu Acher (http://mathieuacher.com) is an Associate Professor at University of Rennes 1, France. His research focuses on reverse engineering, modeling, reasoning, and learning variability in various kinds of artefacts and domains. He is the main developer of FAMILIAR (http:/ / familiar-project.github.io) for which he has designed and implemented novel automated operations. He has authored more than 80 peer-reviewed papers in international journals, conferences or workshops (e.g., ESE, STTT, SCP, ASE, ESEC/FSE, ISSTA, SPLC, CAiSE, FASE, IJCAI). He was PC co-chair of Software Product Line Conference in 2017.

\section{Dr. Jean-Marc Jézéquel}

is a Professor at the University of Rennes and Director of IRISA, one of the largest public research lab in Informatics in France. He is also head of research of the French Cyber-defense Excellence Cluster and the director

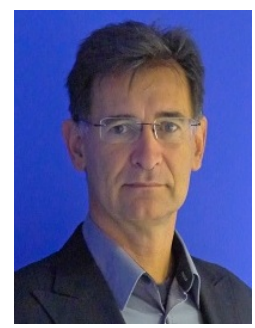
of the Rennes Node of EIT

Digital. In 2016 he received the Silver Medal from CNRS. His interests include model driven software engineering for software product lines, and specifically component based, dynamically adaptable systems with quality of service constraints, including security, reliability, performance, timeliness etc. He is the author of 4 books and of more than 250 publications in international journals and conferences. He was a member of the steering committees of the AOSD and MODELS conference series. He also served on the editorial boards of IEEE Computer, IEEE Transactions on Software Engineering, the Journal on Software and Systems, on the Journal on Software and System Modeling and the Journal of Object Technology. He received an engineering degree from Telecom Bretagne in 1986, and a Ph.D. degree in Computer Science from the University of Rennes, France, in 1989. 


\section{APPENDIX}

\begin{tabular}{|c|c|}
\hline Concepts & Dispersion Score \\
\hline \hline electronic & 0.423 \\
\hline sports & 0.423 \\
\hline animal & 0.404 \\
\hline appliance & 0.365 \\
\hline kitchen & 0.365 \\
\hline person & 0.365 \\
\hline accessory & 0.346 \\
\hline vehicle & 0.346 \\
\hline furniture & 0.327 \\
\hline outdoor & 0.327 \\
\hline food & 0.308 \\
\hline indoor & 0.308 \\
\hline
\end{tabular}

Table 5: Dispersion scores for all concepts in the COCO dataset

\begin{tabular}{|l|c|c|c|c|c|}
\hline Test Cases & Prog. 1 & Prog. 2 & $\ldots$ & Prog. 21 & Dispersion \\
\hline tc 01 & 1.1425 & 93.4543 & $\ldots$ & 1 & 0.143 \\
\hline tc 02 & 15.3550 & 1 & $\ldots$ & 1.4269 & 0.095 \\
\hline & \multicolumn{5}{|c|}{$\ldots$} \\
\hline tc 35 & 1 & 1.0634 & $\ldots$ & 1.5155 & 0.047 \\
\hline & 1.0255 & 18.4676 & $\ldots$ & 1.0117 & 0.095 \\
\hline tc 84 & $\ldots$ \\
\hline
\end{tabular}

Table 6: Sample of Execution time observations regarding generated Haxe programs. (similar table regarding other examples are available in appendixes).

\begin{tabular}{|c|c|c|c|}
\hline Concepts & Initial Perf & Computed Perf & Diff. Perf. \\
\hline \hline Megvii (Face++) & 0.526 & 0.539 & 0.013 \\
\hline bharat_umd & 0.482 & 0.494 & 0.012 \\
\hline IL & 0.420 & 0.430 & 0.010 \\
\hline umd_det & 0.408 & 0.416 & 0.008 \\
\hline Deformable R-FCN & 0.375 & 0.391 & 0.016 \\
\hline HRI & 0.367 & 0.392 & 0.025 \\
\hline Imagine Lab & 0.357 & 0.372 & 0.015 \\
\hline CMU_A2_VGG16 & 0.324 & 0.338 & 0.014 \\
\hline Ttester & 0.294 & 0.312 & 0.018 \\
\hline CMU_A2 & 0.256 & 0.276 & 0.020 \\
\hline drl & 0.235 & 0.247 & 0.012 \\
\hline 1026 & 0.178 & 0.196 & 0.018 \\
\hline IRONYUN & 0.153 & 0.154 & 0.001 \\
\hline
\end{tabular}

Table 7: Excerpt of competitors' performances differences between the original benchmark (Initial Perf) and our reduced set of 5 concepts (Computed Perf)

\begin{tabular}{|c|c|c|}
\hline Techniques' names & Initial Rank & New Rank \\
\hline Megvii (Face++) & 1 & 1 \\
\hline UCenter & 2 & 2 \\
\hline MSRA & 3 & 3 \\
\hline FAIR Mask R-CNN & 4 & 4 \\
\hline Trimps-Soushen+QINIU & 5 & 6 \\
\hline bharat_umd & 6 & 5 \\
\hline DANet & 7 & 7 \\
\hline BUPT-Priv & 8 & 8 \\
\hline DL61 & 9 & 10 \\
\hline DeNet & 10 & 9 \\
\hline IL & 11 & 12 \\
\hline G-RMI & 12 & 11 \\
\hline VCA & 13 & 13 \\
\hline LDL & 14 & 14 \\
\hline PingAn AI Lab & 15 & 15 \\
\hline umd_det & 16 & 16 \\
\hline MSRA_2016 & 17 & 17 \\
\hline DeepInsight & 18 & 19 \\
\hline RetinaNet (1 model) & 19 & 18 \\
\hline DGIST-FATRC & 20 & 21 \\
\hline Deformable R-FCN & 21 & 23 \\
\hline MSRA_2015 & 22 & 20 \\
\hline HRI & 23 & 22 \\
\hline FPN (single model) & 24 & 25 \\
\hline Trimps-Soushen & 25 & 24 \\
\hline Imagine Lab & 26 & 26 \\
\hline mcc_lab & 27 & 28 \\
\hline Wall & 28 & 27 \\
\hline ANLV & 29 & 30 \\
\hline FAIRCNN & 30 & 29 \\
\hline CMU_A2_VGG16 & 31 & 31 \\
\hline DeNet & 32 & 32 \\
\hline ION & 33 & 33 \\
\hline CMU Cylab & 34 & 34 \\
\hline COCO VGG16 Baseline & 35 & 36 \\
\hline Ttester & 36 & 35 \\
\hline ToConcoctPellucid & 37 & 38 \\
\hline MCLAB & 38 & 37 \\
\hline hust-mclab & 39 & 39 \\
\hline MCPRL & 40 & 40 \\
\hline CMU_A2 & 41 & 41 \\
\hline Darknet & 42 & 43 \\
\hline UofA & 43 & 42 \\
\hline FRCNN CNET & 44 & 45 \\
\hline COCO Baseline & 45 & 44 \\
\hline drl & 46 & 46 \\
\hline Decode & 47 & 47 \\
\hline Wall_2015 & 48 & 48 \\
\hline SinicaChen & 49 & 49 \\
\hline UCSD & 50 & 51 \\
\hline 1026 & 51 & 50 \\
\hline iRONYUN & 52 & 52 \\
\hline
\end{tabular}

Table 8: Excerpt of differences in the ranks for the 52 competitors: Initial Rank is computed over the entire dataset while New Rank is computed over the reduced dataset 